\title{
Femtosecond Soft X-ray Spectroscopy of Solvated Transition-Metal Complexes: Deciphering the Interplay of Electronic and Structural Dynamics
}

\author{
Nils Huse, ${ }^{*,+}$ Hana Cho, ${ }^{+, \neq}$Kiryong Hong, ${ }^{\ddagger}$ Lindsey Jamula, ${ }^{\S}$ Frank M. F. de Groot, ${ }^{\perp}$ Tae Kyu Kim, ${ }^{*, \neq}$ \\ James K. McCusker, ${ }^{\S}$ and Robert W. Schoenlein ${ }^{\dagger}$ \\ ${ }^{\dagger}$ Ultrafast X-ray Science Laboratory, Chemical Sciences Division, Lawrence Berkeley National Laboratory, Berkeley, California 94720, \\ United States \\ ${ }^{\ddagger}$ Department of Chemistry and Chemistry Institute of Functional Materials, Pusan National University, Busan 609-735, Republic of Korea \\ ${ }_{\S}^{\S}$ Department of Chemistry, Michigan State University, East Lansing, Michigan 48824, United States \\ ${ }^{\perp}$ Department of Chemistry, Utrecht University, 3584 Utrecht, Netherlands
}

Supporting Information

ABSTRACT: We present the first implementation of femtosecond soft X-ray spectroscopy as an ultrafast direct probe of the excited-state valence orbitals in solution-phase molecules. This method is applied to photoinduced spin crossover of $\left[\mathrm{Fe}\left(\operatorname{tren}(\mathrm{py})_{3}\right)\right]^{2+}$, where the ultrafast spinstate conversion of the metal ion, initiated by metal-to-ligand charge-transfer excitation, is directly measured using the intrinsic spin-state selectivity of the soft X-ray L-edge transitions. Our results provide important experimental data concerning the mechanism of ultrafast spin-state conversion and subsequent electronic and structural dynamics, highlighting the potential of this technique to study ultrafast phenomena in the solution phase.

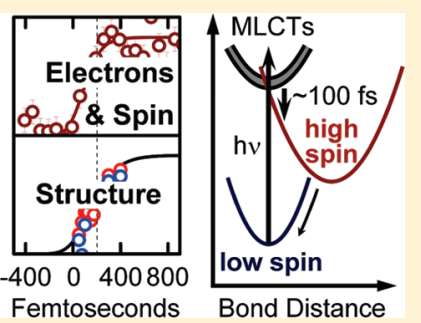

SECTION: Electron Transport, Optical and Electronic Devices, Hard Matter

$\mathrm{T}$ ransition-metal-based spin crossover complexes are a fascinating class of compounds due to their ability to change the spin-state in response to external perturbations such as temperature, pressure, and light. ${ }^{1}$ The dramatic differences in the structural, optical, and magnetic properties between the low- and high-spin forms of such systems has generated interest in their potential application in a variety of molecule-based devices. In this context, it is crucial to understand the interplay of structure and valence charge distributions. Ultrafast optical spectroscopy methods have been successfully used in the past to elucidate details associated with photoinduced spin-state conversion; ${ }^{2-6}$ however, the link between these observables and changes in molecular structure and electronic configuration can often be difficult to establish. Transient core-level spectroscopy and X-ray diffraction provide an attractive route to study the interplay of molecular structure and valence electronic configuration of metal centers in such systems while in the excited state. ${ }^{7-11,26}$ For example, transient X-ray absorption spectroscopy (XAS) at the Fe K-edge of low-spin $\mathrm{Fe}$ (II) complexes has provided detailed information on structural changes (e.g., $\sim 0.2$ A elongation of the $\mathrm{Fe}-\mathrm{N}$ bonds) subsequent to excitation of metal-to-ligand charge-transfer transitions. ${ }^{8-11,26}$

While much insight has been obtained from structural probes, transient K-edge absorption spectroscopy of the important firstrow transition metals delivers limited information about changes in electronic configuration because of the broad and relatively weak spectral features arising from formally dipole-forbidden transitions $(1 \mathrm{~s} \rightarrow 3 \mathrm{~d}) .{ }^{10}$ In contrast, transient L-edge absorption spectroscopy in the soft X-ray range exploits Laporte allowed
$(2 \mathrm{p} \rightarrow 3 \mathrm{~d})$ transitions that directly probe electronic changes associated with valence orbitals (Figure 1A) that dominate the chemistry in these systems. We recently demonstrated the first transient soft X-ray spectroscopy ${ }^{12,13}$ of solvated molecules, which provided a detailed picture of the changes in valence electron distributions in the $3 \mathrm{~d}$ manifold of a low-spin iron(II) complex subsequent to charge-transfer excitation. This type of spectroscopy provides crucial information on photoinduced changes in bonding and electronic configuration ${ }^{13}$ not accessible by other methods. ${ }^{4}$ Our previous picosecond study only allowed characterization of the initial- and final-state valence charge distributions due to the subpicosecond time scale of charge transfer to high-spin relaxation. ${ }^{2,5}$ Extending L-edge spectroscopy of firstrow transition metals to femtosecond time resolution holds great potential for examining changes in valence charge density during the course of photoinduced chemical transformations, but it has never been demonstrated for solution-phase systems due, in part, to the difficulties associated with the application of soft X-ray spectroscopy to liquid samples. ${ }^{15,16,27}$ Herein, we report the first example of such a measurement. Specifically, photoinduced low-spin to high-spin conversion of a six-coordinate $\mathrm{Fe}$ (II) compound has been studied with femtosecond soft $\mathrm{X}$-rays at the $\mathrm{Fe} \mathrm{L}_{3}$-edge, providing a direct measure of changes in the valence orbital configuration concomitant with photoinduced spin crossover.

\section{Received: February 4, 2011}

Accepted: February 24, 2011 

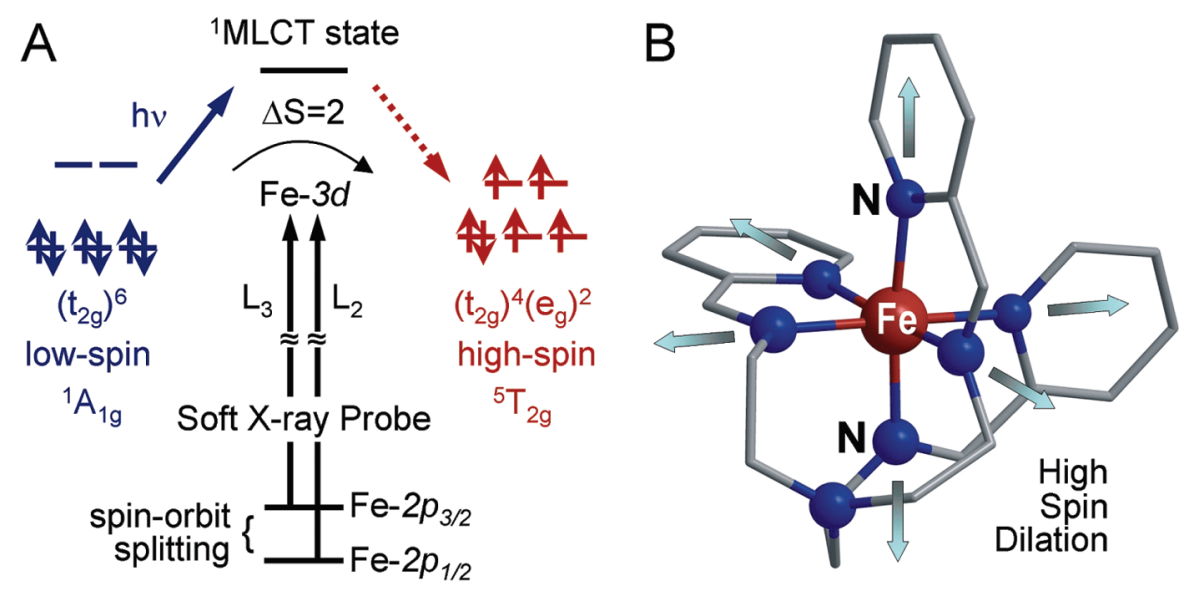

Figure 1. (A) Qualitative orbital diagram for an octahedral Fe(II) complex, illustrating how the photoinitiated spin crossover is probed via metal $2 \mathrm{p} \rightarrow$ $3 \mathrm{~d}$ dipole transitions. (B) Schematic drawing of the molecular structure of $\left[\mathrm{Fe}\left(\operatorname{tren}(\mathrm{py})_{3}\right)\right]^{2+}$. Photoinduced metal-to-ligand charge transfer results in ultrafast structural changes that have been previously characterized by picosecond time-resolved EXAFS measurements. ${ }^{8}$

$\left[\mathrm{Fe}\left(\operatorname{tren}(\mathrm{py})_{3}\right)\right]^{2+}\left(\right.$ where tren $(\mathrm{py})_{3}=\operatorname{tris}(2$-pyridylmethylimino-ethyl)amine) (Figure 1B) represents a prototypical iron-based spin crossover with a low-spin $\left({ }^{1} \mathrm{~A}_{1 \mathrm{~g}}\right)$ ground state that undergoes ultrafast spin-state conversion to a high-spin $\left({ }^{5} \mathrm{~T}_{2 \mathrm{~g}}\right)$ state upon excitation into a singlet metal-to-ligand charge-transfer state $\left({ }^{1} \mathrm{MLCT}, \lambda_{\max }=560 \mathrm{~nm}\right)$. The photoinduced excited-state dynamics of this compound have been studied by a range of experimental techniques including femtosecond electronic absorption spectroscopy and stimulated Raman scattering ${ }^{2,5}$ as well as picosecond EXAFS spectroscopy. ${ }^{8}$ The confluence of the femtosecond laser experiments indicate that the final state is formed on subpicosecond time scales after the initial MLCT absorption; femtosecond soft X-ray spectroscopy is therefore uniquely positioned to examine this process.

Measurements were carried out at the ultrafast X-ray facility of the Advanced Light Source. Briefly, a $100 \mathrm{mM}$ acetonitrile solution of $\left[\mathrm{Fe}\left(\operatorname{tren}(\mathrm{py})_{3}\right)\right]\left(\mathrm{PF}_{6}\right)_{2}$ in a $2 \mu \mathrm{m}$ thick silicon nitride cell was excited by a $560 \mathrm{~nm}$ laser pulse $\left(70 \mathrm{fs}\right.$ duration, $3 \mathrm{~mJ} / \mathrm{cm}^{2}$ incident flux, $0.5 \mathrm{kHz}$ repetition rate). Transmission changes were probed by tunable soft X-ray pulses ( $\sim 10$ photons/pulse/ $0.1 \%$ bandwidth, $200 \mathrm{fs}$ duration) from a slicing source ${ }^{17}$ at a repetition rate of $1 \mathrm{kHz}$. Differential absorption changes at the $\mathrm{Fe}$ $\mathrm{L}_{3}$-edge were recorded by creating interleaved data from the laserexcited and ground-state sample. A schematic of the experimental apparatus can be found in Figure $S 1$ of the Supporting Information.

Figure $2 \mathrm{~A}$ shows a comparison of the differential X-ray absorption spectrum from our picosecond study with our new results recorded with almost 3 orders of magnitude higher temporal resolution. Both differential spectra reveal a shift in the absorption spectrum to lower energies in addition to changes in the overall line shape and an increase in absorption integrated across the $\mathrm{L}_{3}$-edge. More significantly, the strong similarity of the differential absorption spectra at time delays of $700 \mathrm{fs}$ and $90 \mathrm{ps}$ demonstrates the existence of a new core-level transition associated with the excited state formed subsequent to chargetransfer excitation of the chromophore. The $\mathrm{L}_{3}$-edge of the ${ }^{1} \mathrm{~A}_{1 \mathrm{~g}}$ state and transient ${ }^{5} \mathrm{~T}_{2 \mathrm{~g}}$ state reconstructed from the picosecond differential $\mathrm{X}$-ray absorption spectrum is plotted in Figure 2B. For an understanding of these spectra we briefly summarize our previous work ${ }^{13}$ which concluded from comparison of the $\mathrm{L}_{2}$ and $\mathrm{L}_{3}$ absorption line shapes with model systems and multiplet calculations that in the low-spin ${ }^{1} \mathrm{~A}_{1 \mathrm{~g}}$ ground state,

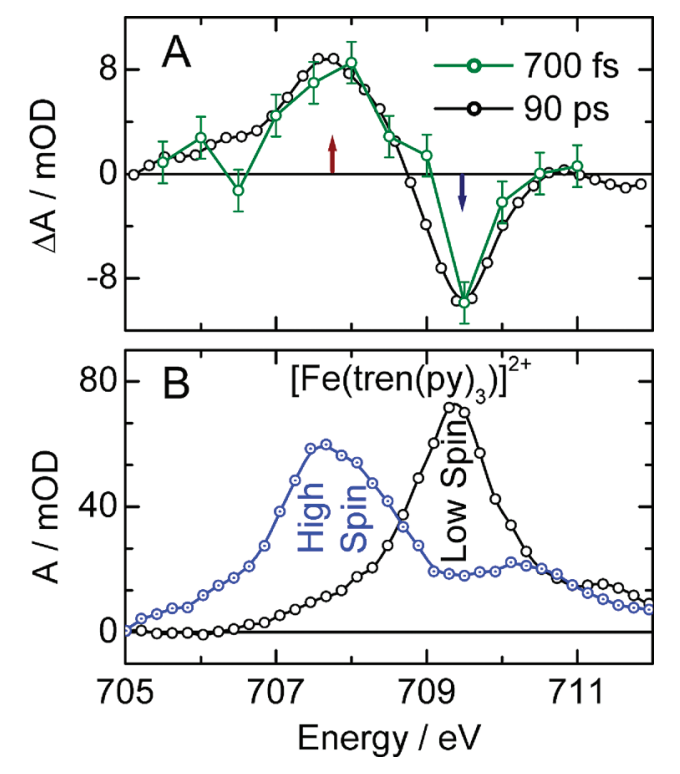

Figure 2. (A) Time-resolved differential X-ray absorption spectra of $\left[\mathrm{Fe}\left(\operatorname{tren}(\mathrm{py})_{3}\right)\right]^{2+}$ in $\mathrm{CH}_{3} \mathrm{CN}$ solution at the $\mathrm{Fe}_{3}$-edge. The red and blue arrows indicate spectral positions at which the time-dependent data shown in Figure 3A were acquired. (B) L-edge spectra of $\left[\mathrm{Fe}\left(\operatorname{tren}(\mathrm{py})_{3}\right)\right]^{2+}$ in $\mathrm{CH}_{3} \mathrm{CN}$ in the low-spin ground state (black) and the transient high-spin excited state (blue) at $\Delta t=90 \mathrm{ps}$.

strong $\pi$-back-bonding results in delocalization of the $\mathrm{Fe} 3 \mathrm{~d}\left(\mathrm{t}_{2 \mathrm{~g}}\right)$ electron into the $\pi^{*}$-orbitals of the tren $(\mathrm{py})_{3}$ ligands. The highspin ${ }^{5} \mathrm{~T}_{2}$ excited state is characterized by suppressed $\pi$-back-bonding and attenuated $\sigma$-donation from the tren $(\text { py })_{3}$ ligand relative to the low-spin ground state. Both effects lead to more localized N 2p and $\mathrm{Fe} 3 \mathrm{~d}$ orbitals while keeping the integrated occupancy of the metalbased $e_{g}$ and $t_{2 g}$ orbitals roughly constant, that is, the overall $\mathrm{Fe} 3 \mathrm{~d}$ charge density is unchanged while the character of the metal-ligand bonding becomes more ionic in nature. ${ }^{13}$

Complementary to the spectral domain, femtosecond absorption changes as a function of time delay have been recorded (Figure 3A). Transients at the maxima of the bleach and absorptive features are both characterized by a steep rise followed by a plateau which extends well beyond 1 ps; no further absorption changes on time scales between a few picoseconds and several nanoseconds 


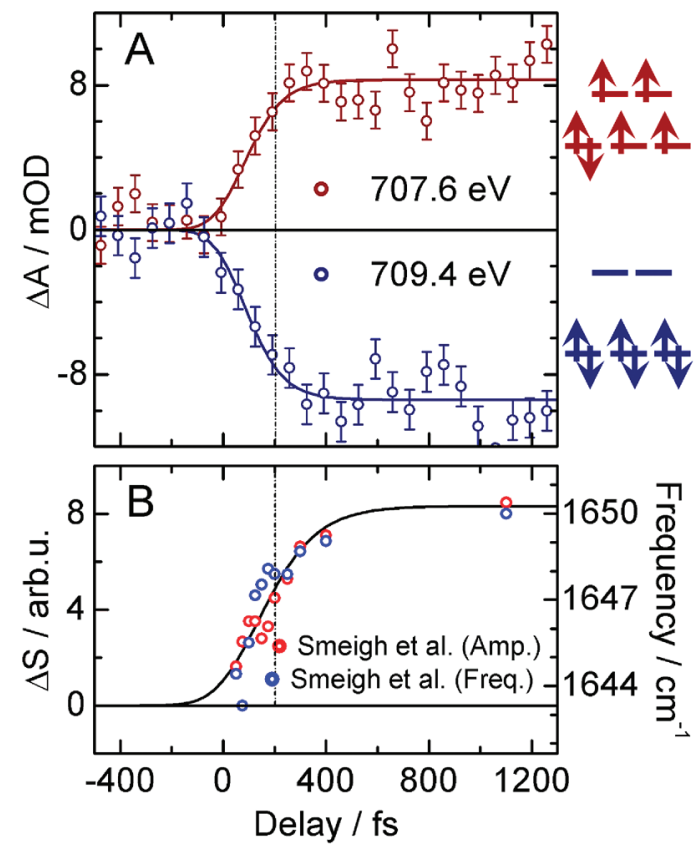

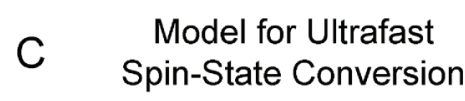

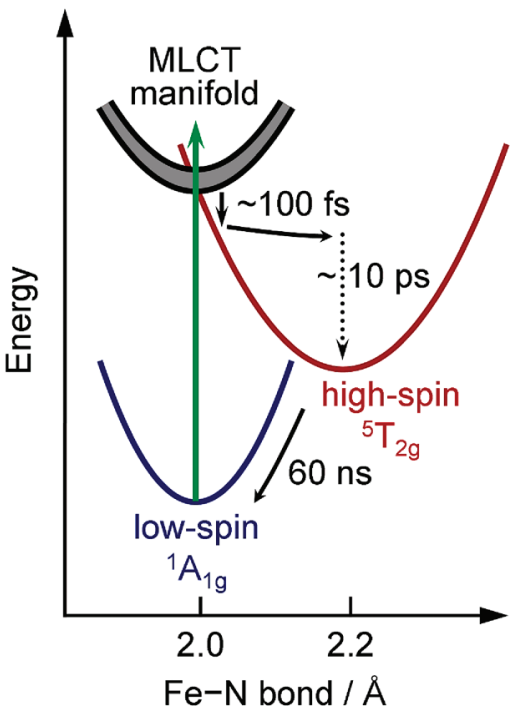

Figure 3. (A) $\mathrm{L}_{3}$-edge absorption changes of $\left[\mathrm{Fe}\left(\operatorname{tren}(\mathrm{py})_{3}\right)\right]^{2+}$ in $\mathrm{CH}_{3} \mathrm{CN}$ solution as a function of pump-probe delay (open circles) at the two spectral positions indicated in Figure 2A. The solid lines correspond to a fit of the data to a model which includes a time constant for the formation of the ${ }^{5} \mathrm{~T}_{2 \mathrm{~g}}$ state and a $60 \mathrm{~ns}$ lifetime for the ${ }^{5} \mathrm{~T}_{2 \mathrm{~g}}$ state. ${ }^{2}$ See the Supporting Information for a more detailed discussion of the kinetic model. (B) Transients acquired from femtosecond stimulated Raman scattering ${ }^{5}$ (FSRS) of the $\mathrm{C}-\mathrm{N}$ stretching mode of $\left[\mathrm{Fe}\left(\operatorname{tren}(\mathrm{py})_{3}\right)\right]^{2+}$ in $\mathrm{CH}_{3} \mathrm{CN}$ solution; the red and blue circles correspond to changes in the amplitude and frequency of the FSRS signal, respectively. The data were fit with the kinetic model used in panel A. (C) Schematic representation of the excited-state evolution of $\left[\mathrm{Fe}\left(\operatorname{tren}(\mathrm{py})_{3}\right)\right]^{2+}$ following charge-transfer excitation based on all of the available data on this compound. The $\sim 10$ ps component corresponds to vibrational cooling in the high-spin state as determined by time-resolved electronic absorption and resonance Raman measurements. ${ }^{2,5}$

were observed, implying that the differential spectra in Figure 2A and hence the changes in spin-state and electronic configuration reported previously ${ }^{13}$ occur within $300 \mathrm{fs}$. Fitting these transients to a three-level rate equation model convolved with the instrumental response function of $200 \mathrm{fs}$ (fwhm) yields a time constant of $85 \pm 75$ fs for the formation time of the high-spin ${ }^{5} \mathrm{~T}_{2 \mathrm{~g}}$ state. This constitutes a unique, direct measurement of photoinduced spin-state conversion in a solvated compound on femtosecond time scales, owing to the intrinsic spin-state selectivity of the $2 \mathrm{p} \rightarrow 3 \mathrm{~d}$ transitions. Specifically, the spin-orbit splitting of the Fe $2 \mathrm{p}$ orbitals renders the number of allowed dipole transitions (and hence the spectral weight) of the $\mathrm{L}_{2}$-edge $\left(2 \mathrm{p}_{1 / 2} \rightarrow 3 \mathrm{~d}\right)$ and the $\mathrm{L}_{3}$-edge $\left(2 \mathrm{p}_{3 / 2} \rightarrow 3 \mathrm{~d}\right)$ dependent on the orbital occupancy of the $3 \mathrm{~d}$ manifold. This in turn defines the configuration from which the electronic term states of the compound derive and, ultimately, the spin-state of the molecule. ${ }^{18}$

Our detailed findings on the ultrafast changes in the metalcentered valence electron distribution can be compared with all of the other ultrafast spectroscopic data that have been acquired on this compound. Transient absorption spectroscopy in the visible and ultraviolet wavelength range have established a time constant of $80 \pm 20$ fs for the depopulation of the MLCT manifold ${ }^{2}$ and the presence of two low-spin/high-spin isosbestic points identical to those determined from steady-state spectroscopy. ${ }^{5}$ The latter measurements placed an upper limit on the time scale for ${ }^{5} \mathrm{~T}_{2 \mathrm{~g}}$ formation of $<250 \mathrm{fs}$, consistent with the data in Figure 3A. The similar time constants for depopulation of the MLCT manifold and population of the ${ }^{5} \mathrm{~T}_{2 \mathrm{~g}}$ state as measured by femtosecond L-edge spectroscopy (Figure $3 \mathrm{~A}$ ) hint at a direct relaxation from the MLCT manifold to the ${ }^{5} \mathrm{~T}_{2 \mathrm{~g}}$ state, as already suggested recently for a similar compound, ${ }^{19}$ although involvement of other excited states in the relaxation process cannot be excluded based on the data in Figure 3A due, in part, to the time resolution of the soft X-ray experiment. ${ }^{20}$

Femtosecond stimulated Raman spectroscopy (FSRS) has been used to probe vibrational changes associated with the photoinduced spin-state conversion in $\left[\mathrm{Fe}\left(\operatorname{tren}(\mathrm{py})_{3}\right)\right]^{2+}$ in solution. ${ }^{5}$ This experiment probed structural dynamics upon photoinduced spin crossover by measuring changes in frequency and amplitude of the $\mathrm{C}-\mathrm{N}$ (imine) stretching mode, establishing a formation time of $<200 \mathrm{fs}$ for the ligand cage dilation associated with the high-spin form. ${ }^{5}$ Figure $3 \mathrm{~B}$ shows a plot of both Raman signatures along with fits to our kinetic model, yielding a formation time of $150 \pm 60 \mathrm{fs}$. It can be seen that all of the data sets can be described with a common model, except for slight differences in the formation time of spectral features associated with the high-spin state (vide infra). In this context, it is important to note that the three types of femtosecond data that we have been discussing-visible absorption, Raman, and soft X-ray absorption-probe different aspects of the electronic and geometric properties of the molecule. We therefore view the remarkable consistency across all of these measurements as strong support for the overall kinetic scheme describing the excitedstate dynamics of this compound (Figure 3C).

Studies of other metal polypyridyl complexes in solution using ultraviolet/visible absorption and fluorescence up-conversion spectroscopy have established very similar time constants for the depopulation of the respective MLCT manifold. ${ }^{3,6}$ The structural changes in these systems were probed with femtosecond infrared $^{4}$ and iron K-edge XANES ${ }^{9}$ spectroscopy, with the latter resembling precisely the kinetics of $\left[\mathrm{Fe}\left(\operatorname{tren}(\mathrm{py})_{3}\right)\right]^{2+}$ that 
underlie the femtosecond Raman data in Figure 3B (see Figure S2 in the Supporting Information). While the ligand cage expansion results in an increase in the polarizability of the compound (and therefore the Raman scattering cross section), ${ }^{5}$ it also changes the spectral interference in the multiscattering region of the iron K-edge XANES spectrum. ${ }^{9}$ Both techniques are therefore sensitive to atomic structure, albeit in different ways. With regard to core-level transitions, we note that the femtosecond XANES study with hard X-rays ${ }^{9}$ is complementary to our femtosecond L-edge study with soft X-rays, ${ }^{13}$ with the former probing changes in atomic structure and the latter probing changes in valence charge density.

A recent theoretical study by de Graaf and Sousa ${ }^{21}$ on $\left[\mathrm{Fe}(\mathrm{bpy})_{3}\right]^{2+}$ (where bpy = bipyridine) concludes that the ${ }^{1}$ MLCT and ${ }^{3}$ MLCT states substantially mix upon photoexcitation, leading to ultrafast initial spin-state conversion in this spin crossover compound while in the charge-transfer manifold. ${ }^{3}$ Moreover, the theoretical calculations predict that the MLCT states' equilibrium position along the $\mathrm{Fe}-\mathrm{N}$ bond coordinate is close to that of the low-spin ground state and that the metalcentered ${ }^{5} \mathrm{~T}_{2 \mathrm{~g}}$ quintet state crosses the MLCT band near this equilibrium geometry. The resulting model as depicted in Figure $3 \mathrm{C}$ is along the lines of a previously proposed mechanism for ultrafast spin crossover in this class of systems. ${ }^{19}$ Initial formation of the MLCT state(s) occurs without substantial ligand cage dilation, followed by depopulation of the charge-transfer manifold to ligand field state(s) with a time constant of $\leq 100 \mathrm{fs}^{2}$ The evolution to the ${ }^{5} \mathrm{~T}_{2 \mathrm{~g}}$ state proceeds with a similar time constant and triggers ultrafast structural dynamics along the $\mathrm{Fe}-\mathrm{N}$ bond coordinate. Subsequent slower structural rearrangements occur on the time scale of $\sim 10$ ps as the compound relaxes further toward the equilibrium structure within the ${ }^{5} \mathrm{~T}_{2 \mathrm{~g}}$ state. ${ }^{2,5}$ This model explains why the electronic/spin-state conversion probed by the L-edge transients in Figure $3 \mathrm{~A}$ could display a shorter time constant than the structural dynamics associated with ligand cage dilation, probed via the FSRS measurements in Figure 3B; the system assumes high-spin ${ }^{5} \mathrm{~T}_{2 \mathrm{~g}}$ character before structural relaxation sets in.

The current model of photoinitiated spin crossover with initial population of the MLCT manifold and subsequent formation of the high-spin state would be best tested by ultrafast L-edge spectroscopy with even higher temporal resolution. In principle, free-electron lasers (FELs) provide X-ray pulses of sufficiently short duration to allow for such measurements. The current timing systems limit the effective resolution of averaged transients to $\geq 200 \mathrm{fs}$, which is comparable to the time resolution of our experiment. However, single-shot experiments could not only exploit the short and intense X-ray pulses provided by X-ray FELs but would serve as a temporal cross-correlation between laser and X-ray pulses. We note that solvation dynamics on a subpicosecond time scale should influence the dynamics that underlie the transients in Figure 3A. This is particularly true while the system is in the charge-transfer manifold, where fluctuating electric forces are more effective in perturbing the excited valence charge distribution that is delocalized across the exposed ligand structure. ${ }^{22}$ We expect the solvent influence on the ligand field states to be much weaker because they are metal-localized and thus sterically and dielectrically shielded by the ligand framework. $^{23}$ A static X-ray absorption spectroscopic study ${ }^{24}$ in fluorescence yield mode has attributed unusual ground-state L-edge line shapes of aqueous $\left[\mathrm{Fe}(\mathrm{bpy})_{3}\right]^{2+}$ to orbital interactions between the iron site and unoccupied oxygen orbitals (however, fluorescence yield XAS signals may be obscured by substantial contributions from solvent absorption and emission as well as diffuse scattering). A systematic solvent study with $<50$ fs time resolution could aid in clarifying the role of the solvent in these excited-state dynamics.

In summary, the work presented herein constitutes the first ultrafast soft X-ray study of a molecule in solution and provides important experimental evidence on the mechanism of photoinduced spin-state conversion. More generally, these results underscore the power of ultrafast soft X-ray absorption spectroscopy for the study of electron and related spin dynamics of solution-phase systems with femtosecond time resolution. We believe that this method opens up a range of future opportunities to investigate ultrafast chemical reactions in molecules as well as functional nanoscopic systems under ambient conditions, providing unprecedented information on spin-states and valence electron distributions not accessible with visible spectroscopy or diffractive techniques. Finally, the prevalence of first-row transition-metal centers in many biological systems ${ }^{25}$ and their important role in catalysis underscores the importance of this method for many other research areas to interrogate the evolution of electronic valence charge distribution.

\section{ASSOCIATED CONTENT}

S Supporting Information. Schematic of the femtosecond $\mathrm{X}$-ray experiment. Compilation of transients acquired from ultrafast structural probes. Four-level rate equation model and initial condition. This material is available free of charge via the Internet at http://pubs.acs.org.

\section{AUTHOR INFORMATION}

\section{Corresponding Author}

*E-mail: nhuse@lbl.gov (N.H.); tkkim@pusan.ac.kr (T.K.K.).

\section{ACKNOWLEDGMENT}

This work was supported by the Director, Office of Science, Office of Basic Energy Sciences, the Chemical Sciences, Geosciences, and Biosciences Division under the Department of Energy, Contract No. DE-AC02-05CH11231 (N.H., H.C., and R.W.S.) and Grant No. DE-FG02-01ER15282 (J.K.M.), as well as the Basic Science Research Program 2009-0068446 and 20100006570 through the National Research Foundation of Korea (NRF) funded by the Ministry of Education, Science and Technology (T.K.K.). The Advanced Light Source is supported by the Director, Office of Science, Office of Basic Energy Sciences, of the U.S. Department of Energy under Contract No. DE-AC02$05 \mathrm{CH} 11231$.

\section{REFERENCES}

(1) Gütlich, P., Goodwin, H. A., Eds. Spin Crossover in Transition Metal Compounds; Springer: Berlin/Heidelberg, Germany, 2004; Vol. 233-235.

(2) Monat, J.; McCusker, J. Femtosecond Excited-State Dynamics of an Iron(II) Polypyridyl Solar Cell Sensitizer Model. J. Am. Chem. Soc. 2000, 122, 4092-4097.

(3) Gawelda, W.; Cannizzo, A.; Pham, V.-T.; van Mourik, F.; Bressler, C.; Chergui, M. Ultrafast Nonadiabatic Dynamics of $\left[\mathrm{Fe}^{\mathrm{II}}(\mathrm{bpy})_{3}\right]^{2+}$ in Solution. J. Am. Chem. Soc. 2007, 129, 8199-8206.

(4) Wolf, M. M. N.; Gross, R.; Schumann, C.; Wolny, J. A.; Schünemann, V.; Dossing, A.; Paulsen, H.; McGarvey, J. J.; Diller, R. 
Sub-Picosecond Time Resolved Infrared Spectroscopy of High-Spin State Formation in $\mathrm{Fe}(\mathrm{II})$ Spin Crossover Complexes. Phys. Chem. Chem. Phys. 2008, 10, 4264-4273.

(5) Smeigh, A. L.; Creelman, M.; Mathies, R. A.; McCusker, J. K. Femtosecond Time-Resolved Optical and Raman Spectroscopy of Photoinduced Spin Crossover: Temporal Resolution of Low-to-High Spin Optical Switching. J. Am. Chem. Soc. 2008, 130, 14105-14107.

(6) Consani, C.; Prémont-Schwarz, M.; ElNahhas, A.; Bressler, C.; van Mourik, F.; Cannizzo, A.; Chergui, M. Vibrational Coherences and Relaxation in the High-Spin State of Aqueous $\left[\mathrm{Fe}^{\mathrm{II}}(\mathrm{bpy})_{3}\right]^{2+}$. Angew. Chem., Int. Ed. 2009, 48, 7184-7187.

(7) Chen, L. X.; Jager, W. J. H.; Jennings, G.; Gosztola, D. J.; Munkholm, A.; Hessler, J. P. Capturing a Photoexcited Molecular Structure through Time-Domain X-ray Absorption Fine Structure. Science 2001, 292, 262-264.

(8) Khalil, M.; Marcus, M.; Smeigh, A.; McCusker, J.; Chong, H.; Schoenlein, R. W. Picosecond X-ray Absorption Spectroscopy of a Photoinduced Iron(II) Spin Crossover Reaction in Solution. J. Phys. Chem. A 2006, 110, 38-44.

(9) Bressler, C.; Milne, C.; Pham, V.-T.; ElNahhas, A.; van der Veen, R. M.; Gawelda, W.; Johnson, S.; Beaud, P.; Grolimund, D.; Kaiser, M.; et al. Femtosecond XANES Study of the Light-Induced Spin Crossover Dynamics in an Iron(II) Complex. Science 2009, 323, 489-492.

(10) Nozawa, S.; Sato, T.; Chollet, M.; Ichiyanagi, K.; Tomita, A.; Fujii, H.; Adachi, S.; Koshihara, S. Direct Probing of Spin State Dynamics Coupled with Electronic and Structural Modifications by Picosecond Time-Resolved XAFS. J. Am. Chem. Soc. 2010, 132, 61-63.

(11) Gawelda, W.; Pham, V.-T.; Benfatto, M.; Zaushitsyn, Y.; Kaiser, M.; Grolimund, D.; Johnson, S. L.; Abela, R.; Hauser, A.; Bressler, C.; et al. Structural Determination of a Short-Lived Excited Iron(II) Complex by Picosecond X-ray Absorption Spectroscopy. Phys. Rev. Lett. 2007, $98,057401$.

(12) Huse, N.; Khalil, M.; Kim, T. K.; Smeigh, A. L.; Jamula, L.; McCusker, J. K.; Schoenlein, R. W. Probing Reaction Dynamics of Transition-Metal Complexes in Solution via Time-Resolved X-ray Spectroscopy. J. Phys. Conf. Ser. 2009, 148, 012043.

(13) Huse, N.; Kim, T. K.; Jamula, L.; McCusker, J. K.; de Groot, F. M.; Schoenlein, R. W. Photo-Induced Spin-State Conversion in Solvated Transition Metal Complexes Probed via Time-Resolved Soft X-ray Spectroscopy. J. Am. Chem. Soc. 2010, 132, 6809-6816. Please note that an erroneous energy calibration has been used in this reference that differs from the one in the present work.

(14) A recent study demonstrated that, in principle, the same information can be accessed with picosecond hard X-ray emission spectroscopy, facilitating sample handling but suffering from low signal and stronger lifetime broadening; see: Vankó, G.; Glatzel, P.; Pham, V.-T.; Abela, R.; Grolimund, D.; Borca, C. N.; Johnson, S. L.; Milne, C. J.; Bressler, C. Picosecond Time-Resolved X-Ray Emission Spectroscopy: Ultrafast Spin-State Determination in an Iron Complex. Angew. Chem., Int. Ed. 2010, 49, 5910-5912.

(15) Huse, N.; Wen, H.; Nordlund, D.; Szilagyi, E.; Daranciang, D.; Miller, T. A.; Nilsson, A.; Schoenlein, R. W.; Lindenberg, A. M. Probing the Hydrogen-Bond Network of Water via Time-Resolved Soft X-ray Spectroscopy. Phys. Chem. Chem. Phys. 2009, 11, 3951-3957.

(16) Huse, N.; Wen, H.; Schoenlein, R. W.; Lindenberg, A. M. Ultrafast Conversions between Hydrogen Bonded Structures in Liquid Water Observed by Femtosecond X-ray Spectroscopy. J. Chem. Phys. 2009, 131, 234505 .

(17) Schoenlein, R. W.; Chattopadhyay, S.; Chong, H. H. W.; Glover, T. E.; Heimann, P. A.; Shank, C. V.; Zholents, A. A.; Zolotorev, M. S. Generation of Femtosecond Pulses of Synchrotron Radiation. Science 2000, 287, 2237-2240.

(18) de Groot, F. M. F.; de Smit, E.; van Schooneveld, M. M.; Aramburo, L. R.; Weckhuysen, B. M. In-situ Scanning Transmission X-Ray Microscopy of Catalytic Solids and Related Nanomaterials. Chem. Phys. Chem. 2010, 11, 951-962.

(19) Cannizzo, A.; Milne, C.; Consani, C.; Gawelda, W.; Bressler, C.; van Mourik, F.; Chergui, M. Light-Induced Spin Ccrossover in Fe(II)-
Based Complexes: The Full Photocycle Unraveled by Ultrafast Optical and X-ray Spectroscopies. Coord. Chem. Rev. 2010, 254, 2677-2686.

(20) For example, four additional ligand field states deriving from the $\left(t_{2 g}\right)^{5}\left(e_{g}\right)^{1}$ configuration, as well as the ${ }^{3} \mathrm{MLCT}$ state $(\mathrm{s})$, energetically lie between the singlet charge-transfer and ${ }^{5} \mathrm{~T}_{2}$ excited states.

(21) de Graaf, C.; Sousa, C. Study of the Light-Induced Spin Crossover Process of the $\left[\mathrm{Fe}^{\mathrm{II}}(\mathrm{bpy})_{3}\right]^{2+}$ Complex. Chem.-Eur. J. 2010 $16,4550-4556$.

(22) Yeh, A. T.; Shank, C. V.; McCusker, J. K. Ultrafast Electron Localization Dynamics Following Photo-Induced Charge Transfer. Science 2000, 289, 935-938.

(23) Daku, L.; Max, L.; Hauser, A. Ab Initio Molecular Dynamics Study of an Aqueous Solution of $\left[\mathrm{Fe}(\text { bpy })_{3}\right] \mathrm{Cl}_{2}$ in the Low-Spin and in the High-Spin States. J. Phys. Chem. Lett. 2010, 1, 1830-1835.

(24) Aziz, E. F.; Hannelore, R.-F.; Lange, K. M.; Bonhommeau, S.; Chergui, M. Charge Transfer to Solvent Identified Using Dark Channel Fluorescence-Yield L-Edge Spectroscopy. Nat. Chem. 2010, 2, 853-857.

(25) Holm, R. H.; Kennepohl, P.; Solomon, E. I. Structural and Functional Aspects of Metal Sites in Biology. Chem. Rev. 1996, 96 2239-2314.

(26) Lorenc, M.; Hébert, J.; Moisan, N.; Trzop, E.; Servol, M.; Buron-Le Cointe, M.; Cailleau, H.; Boillot, M. L.; Pontecorvo, E.; Wulff, M.; Koshihara, S.; Collet, E. Successive Dynamical Steps of Photoinduced Switching of a Molecular Fe(III) Spin-Crossover Material by Time-Resolved X-Ray Diffraction. Phys. Rev. Lett. 2009, 103, 028301.

(27) Wernet, P.; Gavrila, G.; Godehusen, K.; Weniger, C.; Nibbering, E. T. J.; Elsaesser, T.; Eberhardt, W. Ultrafast temperature jump in liquid water studied by a novel infrared pump-x-ray probe technique. Appl. Phys. A: Mater. Sci. Process. 2008, 92, 511-516. 